Barranco Izquierdo, N. \& Guillén Díaz, C. (2017). Efectos de la mediación oral para la resolución de conflictos comunicativos en aulas de inglés de Educación Primaria con presencia de alumnado extranjero. Revista Electrónica Interuniversitaria de Formación del Profesorado, 20(1), 159-172.

\title{
Efectos de la mediación oral para la resolución de conflictos comunicativos en aulas de inglés de Educación Primaria con presencia de alumnado extranjero
}

Natalia Barranco Izquierdo, Carmen Guillén Díaz

Universidad de Valladolid

\section{Resumen}

Las autoras de esta investigación parten de la premisa relativa al estatus particular, potencial y efectivo de la mediación oral como un eje vertebrador de la comunicación durante el proceso de enseñanza/aprendizaje. Dan cuenta -movilizando procedimientos y estrategias propias de la investigación interpretativa-, de las potencialidades de la mediación oral en la resolución o evitación de los conflictos comunicativos que tienen lugar en el discurso de enseñanza/ aprendizaje de inglés, en aulas de Educación Primaria con presencia de alumnado extranjero. Destacan sus efectos en las actividades comunicativas de la lengua planificadas para el aula, en la perspectiva última de la formación del profesorado, a quien corresponde el logro de la integración socioeducativa de este alumnado.

\section{Palabras clave}

Mediación oral; aulas de inglés; alumnado extranjero; Educación Primaria; investigación interpretativa.

\section{Contacto:}

Natalia Barranco Izquierdo, natalia.barranco@uva.es, Facultad de Educación y Trabajo Social. Paseo de Belén, 1. Campus Miguel Delibes, 47011, Valladolid. 


\title{
Effects of oral mediation for solving communicative conflicts in primary ESL classrooms with foreign students
}

\begin{abstract}
The authors of this research focus on the premise of the potential and effective status of oral mediation as a communication backbone in the teaching and learning process. They give account of the potentialities of oral mediation to solve or avoid communicative conflicts that take place in the teaching and learning discourse in Primary Education classrooms with foreign students. It is carried out by mobilizing the procedures and strategies of the interpretive research. Effects of oral mediation in the planned communicative activities are highlighted, with the ultimate purpose of contributing to better teacher training.
\end{abstract}

\section{Keywords}

Oral mediation; ESL classrooms; foreign students; Primary Education; interpretative research.

\section{Introducción}

Con la presencia de alumnado extranjero con lenguas y/o culturas diferentes a las de escolarización, en el aula de inglés lengua extranjera (ILE) de Educación Primaria, el conflicto comunicativo que tiene lugar en toda situación pedagógica es objeto de especial atención (Trujillo Sáez, 2008), a la luz de las concepciones interaccionistas de la comunicación (Martínez Agudo, 2003), y las constructivistas de la cognición (Tascón Trujillo, 2003; Weil-Barais et Resta-Schweitzer, 2008).

Para la solución de este conflicto comunicativo, en el ámbito disciplinar de Didáctica de las Lenguas-Culturas, la mediación oral (Arroyo González, 2004) que se contempla como una actividad comunicativa de la lengua, desvelada en el documento del Consejo de Europa (2001) -Marco común europeo de referencia para las lenguas-, se nos muestra más allá de la simple traducción. Si bien es evidente que, a diferencia de las otras actividades comunicativas de la lengua, como tal no se planifica intencionalmente en las aulas, si cabe ser considerada un eje vertebrador de la comunicación con potencialidades para la resolución o evitación de conflictos comunicativos.

El hecho de otorgar a la mediación oral un mayor alcance en el discurso del docente, conlleva su consideración como parte integrante del perfil competencial profesional -en términos de conocimiento y comprensión, habilidades y destrezas y actitudes- del profesorado de lenguas (Kelly et al., 2004; Llorent García \& López Azuaga, 2012). De forma que, estimando la pertinencia y utilidad de identificar los efectos de la mediación oral en las diferentes actividades comunicativas de la lengua comúnmente desarrolladas en el aula, es importante tomar como referentes las cualidades esenciales de mejora de la calidad educativa promovidas por De Ketele y Gerard (2007), a saber: la eficacia, la eficiencia y la pertinencia. Para el caso que nos ocupa, nos referimos a eficacia en el sentido de la capacidad de lograr los objetivos planificados por el profesorado; a eficiencia en el sentido de disposición de los recursos necesarios para el logro de dichos objetivos, siendo más 
eficiente cuando se obtienen mejores resultados con menos recursos; y a pertinencia en el sentido de adecuación y conveniencia de los recursos empleados.

\section{Objetivos}

A partir de estas consideraciones, los objetivos que nos hemos planteado en este trabajo son los siguientes:

- Identificar los efectos de la mediación oral en la resolución de conflictos comunicativos en aulas de ILE con presencia de alumnado extranjero.

- Aportar implicaciones metodológicas para la formación del profesorado, respecto al uso de la mediación oral en la resolución de conflictos comunicativos en aulas de ILE con presencia de alumnado extranjero.

\section{Metodología}

Nuestro posicionamiento metodológico en función de los objetivos planteados nos determina a optar por el método de análisis de contenido (Bardin, 1986; Krippendorff, 1990). El carácter esencialmente descriptivo de esta técnica global de indagación nos permite la identificación objetiva de la realidad empírica y, desde su diseño vertical, nos permite descripciones más amplias de las informaciones textuales obtenidas, por la observación de las interacciones de aula fuertemente contextualizadas.

\section{Determinación del contexto de indagación y participantes}

Los criterios fundamentales para la concreción del contexto de indagación y de los participantes en este trabajo de investigación se refieren a cinco condicionantes primordiales:

a) Centros públicos o concertados de Educación Infantil y Educación Primaria en contexto socio-cultural y educativo de amplia tradición monolingüe.

b) Aulas con presencia de alumnado extranjero y de diversos niveles de la Educación Primaria.

c) Alumnado extranjero con presencia estable en el grupo y asistencia continuada como requieren los procedimientos de observación movilizados.

d) Acceso al centro facilitado en primera instancia y alto grado de disponibilidad por parte del profesorado responsable.

e) Heterogeneidad máxima relativa a los años de experiencia docente.

Adecuándonos a estos criterios, seleccionamos para llevar a cabo la observación propiamente dicha, cuatro aulas ubicadas en centros educativos de Valladolid capital y provincia, Palencia capital y Salamanca capital. Para preservar el anonimato, asignamos una letra a cada aula, comenzando por la "Z": AULA Z, AULA Y, AULA X, AULA W. En este sentido, los profesores son nombrados con la letra del aula en la que imparten sus enseñanzas y el alumnado extranjero es nombrado con la letra del aula a la que asisten y un número correlativo empezando por 1. Los participantes se distribuyen según se indica en el Cuadro 1. 


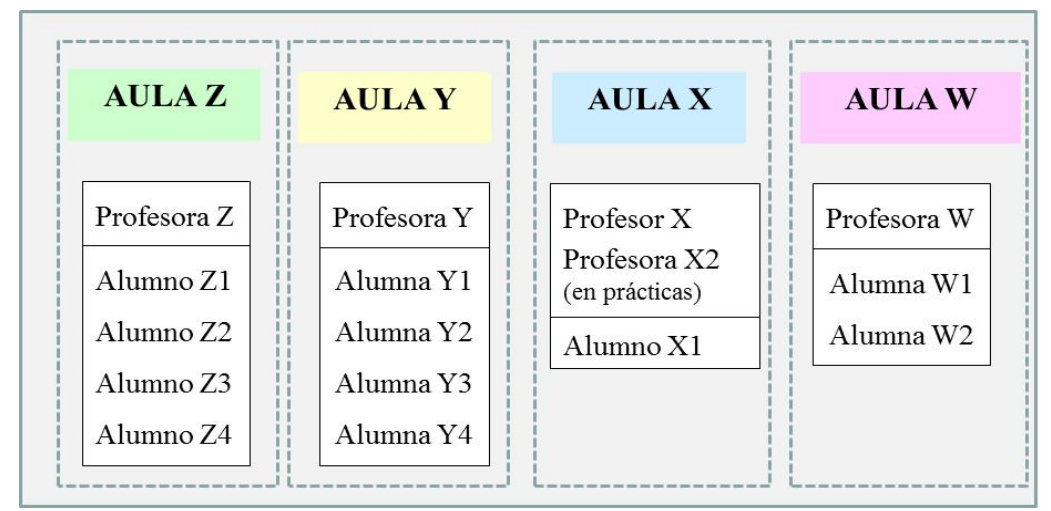

Cuadro 1. Codificación de las aulas y de los participantes objeto de observación.

\section{Datos sociodemográficos por aula y perfiles académicos}

\section{El Aula Z}

La Profesora Z presenta un alto grado de disponibilidad desde la primera toma de contacto, si bien nos muestra vacilaciones sobre si su trabajo va a ser de utilidad para nuestra investigación, argumentando ser una profesora novel que está en su primer año de ejercicio docente. Se muestra siempre muy atenta a lo que sucede en el aula, procurando que todo el alumnado siga de manera normalizada el ritmo de la clase. Usa gestos, realiza dibujos en la pizarra, pone ejemplos o, en última instancia, traduce lo que no se ha comprendido.

El Alumno Z1, que es de República Dominicana, es un alumno muy poco participativo. Rasgo patente incluso en las actividades de interactividad más intensa. Nunca es voluntario, ni responde de manera espontánea. Es un alumno muy introvertido e indeciso.

El Alumno Z2, que es de Ecuador, es un alumno muy participativo que, de manera voluntaria y espontánea, ayuda y es ayudado por sus compañeros en la realización de las actividades. Es un alumno extrovertido y emprendedor.

El Alumno Z3, que también es de Ecuador, participa de manera voluntaria y espontánea. Es un alumno extrovertido y emprendedor.

El Alumno Z4, que es de Colombia, se incorpora a clase con unos días de retraso después de las vacaciones de Navidad. No participa de manera voluntaria ni espontánea en el desarrollo de las actividades, a causa ante todo de su desfase curricular. Aun así, se manifiesta como un alumno extrovertido, si bien, indeciso en sus actuaciones.

\section{El Aula Y}

La Profesora Y, en un primer momento, se muestra reticente a nuestra entrada en el aula, ya que, según comenta, pasó por una experiencia parecida no satisfactoria. Una vez tuvo conocimiento de nuestros propósitos estuvo dispuesta a colaborar. Salvada la reticencia inicial, su grado de disponibilidad fue muy alto. Tiene una amplia experiencia como profesora de ILE en Educación Primaria, ya que lleva 25 años desenvolviendo esta profesión. Su característica más destacable es que, de forma continua, promueve una educación en valores. Declara que trata de fomentar en sus alumnos la tolerancia, el respeto y la empatía. A menudo utiliza estímulos verbales para que éstos se comuniquen en ILE, aunque no siempre lo logra.

La Alumna Y1, que es de República Dominicana, participa exclusivamente cuando se le solicita que lo haga, nunca de manera voluntaria, aunque se mantiene siempre atenta a 
todo lo que dice la Profesora $\mathrm{Y}$ y siempre realiza las actividades demandadas. Es una alumna introvertida y poco emprendedora.

La Alumna Y2, que es de Argelia, participa por norma general de manera voluntaria y espontánea, aunque hay momentos en que los parece estar ausente. En su relación con los demás no hay ningún factor destacable.

La Alumna $Y_{3}$, que es de Ecuador, raramente participa de manera voluntaria y espontánea, aunque siempre que le preguntan sabe responder de forma apropiada. Es una alumna muy introvertida.

La Alumna Y4, que es de Colombia, es la menos participativa de las cuatro y siempre que lo hace es de manera forzosa y controlada. En una sesión de clase comenta incluso que el ILE es una lengua muy difícil. Es una alumna introvertida e indecisa.

\section{El Aula X}

El Profesor X presenta un grado de disponibilidad bastante alto desde la primera entrevista mantenida. Cuenta con una experiencia media y enseña en Educación Primaria en el mismo colegio desde hace 13 años, cuando empezó su trayectoria profesional. Hace uso de gestos y pone ejemplos. Evita la traducción. Coincidimos en una sesión con la Profesora X2 (en situación de prácticas) con la que excepcionalmente mediamos alguna palabra y de la que percibimos en sus actuaciones una disposición muy positiva hacia los alumnos.

El Alumno X1, que es de Marruecos, lleva dos años en España y aún muestra dificultades con el español. En clase siempre se muestra muy participativo de manera voluntaria y espontánea, aunque rara vez conoce la respuesta correcta. Es un alumno extrovertido y receptivo a las ayudas que le ofrecen tanto sus compañeros como el Profesor $X$.

\section{El Aula W}

La Profesora W presenta un alto grado de disponibilidad. Tiene una experiencia de siete años ejerciendo de profesora de inglés como lengua materna en Irlanda (su país de origen) y de dos años como profesora de ILE en nuestro país. La Profesora W se muestra muy cercana al alumnado y trata de que éste se sienta implicado en el proceso de enseñanza/ aprendizaje. Siempre utiliza ILE, tanto con el alumnado en las intervenciones de aula como con la investigadora en las interacciones en diferido. La peculiaridad de esta aula es que corresponde a un centro acogido al convenio entre el Ministerio de Educación y Ciencia y el British Council, por el que se desarrolla un programa bilingüe mediante la impartición de currículo integrado hispano-británico. El alumnado que sigue este programa bilingüe posee una competencia comunicativa en ILE muy elevada si comparamos con el alumnado que asiste a las Aulas $\mathrm{Z}, \mathrm{X}$ e $\mathrm{Y}$ observadas, pertenecientes a centros que no poseen este convenio.

La Alumna W1, que es de Bulgaria, participa de manera voluntaria y espontánea, y se enfada consigo misma cuando los resultados no son tal y como esperaba. Según comenta la Profesora W, es una alumna muy aventajada con respecto al grupo, tanto en ILE como en el resto de las áreas curriculares. Es una alumna extrovertida y emprendedora que colabora con sus compañeros si éstos lo necesitan, especialmente con la Alumna W2.

La Alumna W2, que también es de Bulgaria, no participa de manera voluntaria ni espontánea por no comprender las explicaciones de la profesora o no saber resolver las actividades de forma satisfactoria. Es una alumna extrovertida e indecisa, muy receptiva a las ayudas externas, tanto por parte de la Profesora $\mathrm{W}$ como del resto de sus compañeros, especialmente de la Alumna W1. 


\section{Para la obtención de los datos}

En coherencia con los objetivos planteados y ante las características y particularidades metodológicas que Bisquerra (1989) nos aporta en el marco del método de análisis de contenido, nos inscribimos en:

- un enfoque inductivo, ya que se trata de una muestra poblacional reducida que se puede analizar con profundidad, y las conclusiones se podrían aplicar a contextos similares;

- un enfoque idiográfico, dado que con esta investigación no se pretende establecer leyes generales, sino comprender y describir un fenómeno en particular; y consecuentemente;

- un enfoque descriptivo, ya que abordamos los fenómenos tal cual aparecen en su realidad presente, es decir, que interpretamos lo que es, no lo que fue o lo que será.

Nuestra entrada en las aulas se estructura y organiza, por una parte, previa entrevista con el profesorado participante, y por otra, en función del desarrollo de una unidad didáctica, articulada en un conjunto de actividades planificadas con objetivos y contenidos interconectados e interrelacionados. Se singularizan éstas, en torno a las destrezas de expresión oral y escrita y comprensión oral y escrita. Esta elección delimita el tiempo de estancia en el aula a seis o siete sesiones, y nos permite observar la secuencia completa de dichas actividades.

Podemos así disponer de un corpus de datos provenientes de las interacciones discursivas profesor-alumno, alumno-alumno. Al pretender garantizar la mayor naturalidad y espontaneidad posible de las interacciones, su registro audio y manual (cuaderno de la investigadora) se realiza en una dinámica de observación no participante en el curso del desarrollo de las sesiones.

De cada sesión [codificada como S: seguida del número de orden correspondiente] se registran aquellos elementos discursivos verbales o lingüísticos, y no verbales o no lingüísticos a los que denominamos unidades de mediación o réplicas [cada una codificada como R: seguida del número de orden correspondiente]. Se trata de acontecimientos de aula tales como lo son efectivamente los intercambios discursivos, junto a los gestos, las acciones paralingüísticas y las características paratextuales (Consejo de Europa, 2001), que tienen el valor de efectos en la resolución o evitación de los conflictos comunicativos.

\section{Elementos organizativos para el análisis de los datos}

Se aplica un tratamiento manual que facilita la socialización de los datos y la valoración de la importancia cuantitativa o cualitativa de algunos aspectos (Legendre, 2005). Se analizan e interpretan las réplicas [R:] que, por su complementariedad e interdependencia, sean indicadoras de las cualidades esenciales de eficacia, eficiencia y pertinencia de forma íntimamente relacionadas.

Como está indicado más arriba, tomamos la cualidad esencial de eficacia en el sentido de la capacidad de lograr el efecto que se espera o se desea tras la aplicación de acciones unidades de mediación o réplicas- orientadas a resolver el problema. Según lo cual, y para el caso que nos ocupa, podemos afirmar que una réplica es eficaz a través de los rasgos a) y b):

a) Si se resuelve de manera satisfactoria el conflicto comunicativo. 
b) Si se evita que surja el conflicto comunicativo.

Tomamos la cualidad esencial de eficiencia en el sentido de utilización óptima de esas unidades de mediación o réplicas para alcanzar el mayor grado de eficacia, siendo más eficiente cuantos menos recursos se hayan empleado. Según lo cual y para el caso que nos ocupa, podemos afirmar que una réplica es eficiente a través de los rasgos c) y d):

c) Si se han necesitado tres o menos de tres réplicas para la resolución del conflicto comunicativo.

d) Si al menos una de las réplicas está en ILE.

Tomamos la cualidad esencial de pertinencia en el sentido de utilización adecuada y conveniente de esas unidades de mediación o réplicas para el logro del efecto deseado. Según lo cual y para el caso que nos ocupa, podemos afirmar que una réplica es pertinente a través del rasgo e):

e) Si hay una relación lógica entre los elementos por univocidad, no dando lugar a interpretaciones diferentes, elementos que pueden ser: sólo oral; oral + escrito; sólo gestual; oral + gestual; oral + paralingüístico; oral + paratextual.

Para la presentación y organización del análisis de las réplicas que dan cuenta de los efectos de la mediación oral ante los conflictos comunicativos que surgen en la realización de las actividades comunicativas de la lengua planificadas, se decide confeccionar una tabla para cada aula: Tabla 1 Aula Z; Tabla 2 Aula Y; Tabla 3 Aula X; Tabla 4 Aula W.

En sus columnas y filas se recogen las siguientes referencias significativas y productivas para esta investigación: - los Tipos de Actividades comunicativas de la lengua que se han movilizado durante el desarrollo de la unidad didáctica; - la frecuencia de cada Actividad comunicativa de la lengua y el tiempo global empleado en cada una, expresado en minutos. En la última columna a la derecha, se muestran los rasgos identificativos de cada réplica y su comportamiento en la resolución de conflictos. Se presentan codificadas tal y como mostramos a continuación:

- Si es una sola réplica la que resuelve el conflicto comunicativo, se codifica como (res).

- Si es una sola réplica la que evita el conflicto comunicativo, se codifica como (evi).

- Si es una sucesión de réplicas sin interrupción la que resuelve el conflicto comunicativo, se presenta la última -señalando que forma parte de un grupo de réplicas-, y se codifica como (suc-res).

- Si es una sucesión de réplicas sin interrupción la que evita el conflicto comunicativo, se presenta la última -señalando que forma parte de un grupo-, y se codifica como (sucevi).

- Si la réplica es sólo oral, se codifica como (or).

- Si la réplica es oral + escrito, se codifica como (ores).

- Si la réplica es sólo gestual, se codifica como (ge).

- Si la réplica es oral + gestual, se codifica como (og).

- Si la réplica es oral + paralingüístico, se codifica como (oling).

- Si la réplica es oral + paratextual, se codifica como (otex).

- Si la réplica es efectuada por un profesor que moviliza la mediación y que actúa como Agente mediador, se codifica como (pro).

- Si la réplica es efectuada por un alumno extranjero que moviliza la mediación y que actúa como Agente mediador, se codifica como (alex).

- Si la réplica es efectuada por un alumno que no es extranjero que moviliza la mediación y que actúa como Agente mediador, se codifica como (alum). 
- Si la réplica es efectuada por otro Agente mediador -ni profesorado ni alumnado- que moviliza la mediación, se codifica como (otro).

Las réplicas de las que damos cuenta en este trabajo son aquellas en las que existen indicadores de movilización de mediación oral que favorezcan, en mayor o menor medida, la resolución o, en su caso, evitación de los conflictos comunicativos.

\section{Análisis de los datos}

En las Tablas 1, 2, 3 y 4 que siguen, presentamos el resultado del análisis llevado a cabo en el Aula $Z$, Aula $Y$, Aula $X$ y Aula $W$ respectivamente, atendiendo a las réplicas eficaces, eficientes y pertinentes que promueven la mediación oral para la resolución o evitación de los conflictos comunicativos en las actividades comunicativas de la lengua planificadas de: Expresión oral (EO) -28; Comprensión auditiva (CA) -14; Expresión escrita (EE) -10; Comprensión de lectura (CL) -4; Comprensión audiovisual (CAv) -3; e Interacción oral (IO) -5.

Tabla 1.

Réplicas eficaces, eficientes, pertinentes para la resolución o evitación del conflicto comunicativo- Aula Z

\begin{tabular}{|c|c|c|c|}
\hline $\begin{array}{c}\text { Tipos de Actividad } \\
\text { comunicativa de la } \\
\text { lengua }\end{array}$ & Frecuencia & $\begin{array}{c}\text { Tiempo } \\
\text { global en } \\
\text { minutos(') }\end{array}$ & $\begin{array}{c}\text { Réplica } \\
\text { Eficaz + Eficiente + Pertinente }\end{array}$ \\
\hline $\begin{array}{l}\text { Expresión oral } \\
\text { (EO) }\end{array}$ & 17 & $202^{\prime}$ & $\begin{array}{l}\text { [S:1; R: 12](suc-res-og-pro) } \\
\text { [S: } 1 ; \mathrm{R}: 14] \text { (evi-or-pro) } \\
\text { [S: } 1 ; \mathrm{R}: 47] \text { (evi-or-pro) } \\
\text { [S: } 2 ; \mathrm{R}: 5] \text { (evi-or-pro) } \\
\text { [S: } 2 ; \mathrm{R}: 10] \text { (suc-evi-or-pro) } \\
\text { [S: } 2 ; \mathrm{R}: 22] \text { (suc-res-or-pro) } \\
\text { [S: } 2 ; \mathrm{R}: 25] \text { (evi-or-pro) } \\
\text { [S: 3; R: 31] (evi-oling-pro) } \\
\text { [S: 4; R: 7] (suc-res-or-alum) } \\
\text { [S: 4; R: 28] (evi-or-pro) } \\
\text { [S: 6; R: 21] (res-or-alum) } \\
\text { [S: 6; R: 27] (evi-or-alum) } \\
\text { [S: 6; R: 43] (suc-res-or-pro) } \\
\text { [S: 7; R: } 9] \text { (suc-res-oling-pro) }\end{array}$ \\
\hline $\begin{array}{l}\text { Comprensión } \\
\text { auditiva } \\
\text { (CA) }\end{array}$ & 5 & $115^{\prime}$ & $\begin{array}{l}\text { [S: 1; R: 32] (evi-og-pro) } \\
\text { [S: } 1 ; \mathrm{R}: 33] \text { (evi-or-alex) } \\
\text { [S: } 1 ; \mathrm{R}: 35] \text { (evi-or-pro) } \\
\text { [S: } 1 ; \mathrm{R}: 37 \text { ] (res-oling-pro) } \\
\text { [S: } 1 ; \mathrm{R}: 39] \text { (evi-or-pro) } \\
\text { [S: 1; R: 32] (evi-og-pro) } \\
\text { [S: 2; R: 28] (suc-res-ge-alum) } \\
\text { [S: 4; R: 20] (evi-or-pro) } \\
\text { [S: 7; R: 27] (suc-res-oling-pro) } \\
\text { [S: 7; R: 36] (suc-res-ores-pro) }\end{array}$ \\
\hline $\begin{array}{l}\text { Expresión escrita } \\
\text { (EE) }\end{array}$ & 1 & $23^{\prime}$ & [S: 3; R: 18] (suc-res-og-pro) \\
\hline $\begin{array}{l}\text { Comprensión de } \\
\text { lectura } \\
\text { (CL) }\end{array}$ & 1 & $10^{\prime}$ & - \\
\hline
\end{tabular}




\begin{tabular}{|c|c|c|c|}
\hline $\begin{array}{c}\text { Interacción oral } \\
(\mathrm{IO})\end{array}$ & 1 & $10^{\prime}$ & - \\
\hline
\end{tabular}

Tabla 2.

Réplicas eficaces, eficientes, pertinentes para la resolución o evitación del conflicto comunicativo- Aula Y

\begin{tabular}{|c|c|c|c|}
\hline $\begin{array}{c}\text { Tipos de Actividad } \\
\text { comunicativa de la } \\
\text { lengua }\end{array}$ & Frecuencia & $\begin{array}{c}\text { Tiempo } \\
\text { global en } \\
\text { minutos(') }\end{array}$ & $\begin{array}{c}\text { Réplica } \\
\text { Eficaz + Eficiente + Pertinente }\end{array}$ \\
\hline $\begin{array}{c}\text { Comprensión } \\
\text { audiovisual } \\
\text { (CAv) }\end{array}$ & 3 & $120^{\prime}$ & $\begin{array}{l}\text { [S: 9; R: 15](res-or-pro) } \\
\text { [S: 9; R: 21](res-ge-pro) } \\
\text { [S: 11; R: 7](res-oling-pro) } \\
\text { [S: 11; R: 10](evi-or-pro) } \\
\text { [S: 11; R: 21](res-or-pro) } \\
\text { [S: 13; R: 7](suc-res-or-pro) } \\
\end{array}$ \\
\hline $\begin{array}{l}\text { Expresión escrita } \\
\text { (EE) }\end{array}$ & 6 & $185^{\prime}$ & 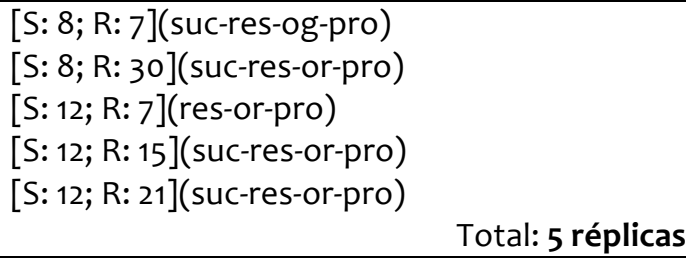 \\
\hline $\begin{array}{l}\text { Expresión oral } \\
\text { (EO) }\end{array}$ & 3 & $50^{\prime}$ & - \\
\hline $\begin{array}{l}\text { Comprensión } \\
\text { auditiva } \\
\text { (CA) }\end{array}$ & 1 & $10^{\prime}$ & - \\
\hline $\begin{array}{l}\text { Interacción oral } \\
(\mathrm{IO})\end{array}$ & 1 & $5^{\prime}$ & - \\
\hline
\end{tabular}

Tabla 3.

Réplicas eficaces, eficientes, pertinentes para la resolución o evitación del conflicto comunicativo- Aula X

\begin{tabular}{|c|c|c|c|}
\hline $\begin{array}{l}\text { Tipos de Actividad } \\
\text { comunicativa de la } \\
\text { lengua }\end{array}$ & Frecuencia & $\begin{array}{c}\text { Tiempo } \\
\text { global en } \\
\text { minutos(') }\end{array}$ & $\begin{array}{c}\text { Réplica } \\
\text { Eficaz + Eficiente+ Pertinente }\end{array}$ \\
\hline $\begin{array}{l}\text { Expresión oral } \\
\text { (EO) }\end{array}$ & 9 & $205^{\prime}$ & $\begin{array}{l}\text { [S: 14; R: 9](suc-res-oling-pro) } \\
\text { [S: 14; R: 24](suc-res-or-pro) } \\
\text { [S: 14; R: 26](evi-or-pro) } \\
\text { [S: 15; R: 17](suc-res-or-pro) } \\
\text { [S: 16; R: 11](suc-res-og-pro) } \\
\text { [S: 17; R: 32](suc-res-or-alum) } \\
\text { [S: 19; R: 6](evi-or-alum) } \\
\text { [S: 19; R: 9](evi-or-alum) } \quad \text { Total: } 8 \text { réplicas }\end{array}$ \\
\hline $\begin{array}{l}\text { Comprensión } \\
\text { auditiva } \\
\text { (CA) }\end{array}$ & 8 & $160^{\prime}$ & $\begin{array}{l}\text { [S: 15; R: 8](evi-og-pro) } \\
\text { [S: 15; R: 28](suc-res-og-pro) } \\
\text { [S: 16; R: 21](suc-res-or-alum) } \\
\text { [S: 14; R: 28](res-or-pro) } \\
\text { [S: 17; R: } 1 \text { ] (evi-or-pro) } \\
\text { [S: 17; R: 20](suc-res-or-pro) } \\
\text { [S: 18; R: 12](suc-res-oling-pro) } \\
\text { [S: 19; R: 17](suc-res-or-pro) }\end{array}$ \\
\hline
\end{tabular}


Tabla 4.

Réplicas eficaces, eficientes, pertinentes para la resolución o evitación del conflicto comunicativo-Aula W

\begin{tabular}{|c|c|c|c|}
\hline $\begin{array}{l}\text { Tipos de Actividad } \\
\text { comunicativa de la } \\
\text { lengua }\end{array}$ & Frecuencia & $\begin{array}{c}\text { Tiempo } \\
\text { global en } \\
\text { minutos(') }\end{array}$ & $\begin{array}{c}\text { Réplica } \\
\text { Eficaz + Eficiente+ Pertinente }\end{array}$ \\
\hline $\begin{array}{l}\text { Expresión oral } \\
\text { (EO) }\end{array}$ & 2 & $45^{\prime}$ & $\begin{array}{l}\text { [S: 20; R: 25](evi-or-pro) } \\
\text { [S: 21; R: 3](res-or-pro) } \\
\text { [S: 21; R: 5](res-oling-otro) } \\
\text { [S: 21; R: 9](suc-res-or-pro) }\end{array}$ \\
\hline $\begin{array}{l}\text { Expresión escrita } \\
\text { (EE) }\end{array}$ & 3 & $80^{\prime}$ & $\begin{array}{l}\text { [S: 20; R: 12](suc-res-or-alex) } \\
\text { [S: 20; R: 22](suc-res-or-alex) } \\
\text { [S: 21; R: 31](suc-res-or-otro) } \\
\text { [S: 22; R: 39](res-or-alex) } \quad \text { Total: } 4 \text { réplicas }\end{array}$ \\
\hline $\begin{array}{l}\text { Comprensión de } \\
\text { lectura } \\
(\mathrm{CL})\end{array}$ & 3 & $70^{\prime}$ & $\begin{array}{l}\text { [S: 24; R: 17](evi-or-alex) } \\
\text { [S: 24; R: 31](suc-res-og-pro) } \\
\text { [S: 25; R: 3](res-or-pro) } \\
\text { [S: 25; R: 9](suc-res-or-alex) } \\
\text { [S: 23; R: 27](suc-res-or-pro) } \\
\text { [S: 25; R: 39](suc-res-or-alum) } \\
\\
\end{array}$ \\
\hline $\begin{array}{l}\text { Interacción oral } \\
\qquad(\mathrm{IO})\end{array}$ & 3 & $105^{\prime}$ & $\begin{array}{l}\text { [S: 22; R: 1](evi-or-pro) } \\
\text { [S: 22; R: 30](suc-res-or-alum) } \\
\\
\quad \text { Total: } 2 \text { réplicas }\end{array}$ \\
\hline
\end{tabular}

Ante estos resultados del análisis, en la Tabla 5, ofrecemos aquellos aspectos cuantitativos sobre los que aplicamos una valoración cualitativa. Se trata de:

- en la primera columna de la izquierda, la cantidad total de réplicas por Aula y por Tipos de Actividades comunicativas de la lengua;

- en la columna central, la cantidad total de réplicas eficaces, eficientes y pertinentes para la resolución o evitación del conflicto comunicativo por Aula y por Tipos de Actividades comunicativas de la lengua; y

- en la columna de la derecha, la distribución porcentual [\%] de réplicas eficaces, eficientes y pertinentes para la resolución o evitación del conflicto comunicativo, respecto del total y por Tipos de Actividades comunicativas de la lengua.

Tabla 5 .

Réplicas eficaces, eficientes, pertinentes respecto del total. Su distribución por actividades comunicativas de la lengua

\begin{tabular}{|c|c|c|c|}
\hline$己$ & $\begin{array}{c}\mathrm{N}^{\circ} \text { total de réplicas } \\
\text { (unidades de mediación) }\end{array}$ & $\begin{array}{c}\mathrm{N}^{\circ} \text { total de réplicas } \\
\text { eficaces/ eficientes/ } \\
\text { pertinentes }\end{array}$ & $\begin{array}{c}{[\%] \text { de réplicas eficaces/ }} \\
\text { eficientes/pertinentes } \\
\text { respecto del total }\end{array}$ \\
\hline
\end{tabular}




\begin{tabular}{|c|c|c|c|c|c|c|c|c|c|c|c|c|c|c|c|}
\hline $\mathrm{Z}$ & \multicolumn{5}{|c|}{147} & \multicolumn{5}{|c|}{25} & \multicolumn{5}{|c|}{$17 \%$} \\
\hline $\mathrm{Y}$ & \multicolumn{5}{|c|}{54} & \multicolumn{5}{|c|}{11} & \multicolumn{5}{|c|}{$20 \%$} \\
\hline$X$ & \multicolumn{5}{|c|}{49} & \multicolumn{5}{|c|}{16} & \multicolumn{5}{|c|}{$32 \%$} \\
\hline W & \multicolumn{5}{|c|}{55} & \multicolumn{5}{|c|}{16} & \multicolumn{5}{|c|}{$29 \%$} \\
\hline$\frac{\mathbb{D}}{\stackrel{D}{D}}$ & \multicolumn{5}{|c|}{$\begin{array}{l}\text { No total de réplicas por Tipos } \\
\text { de Actividades } \\
\text { comunicativas de la lengua }\end{array}$} & \multicolumn{5}{|c|}{$\begin{array}{l}\mathrm{N}^{\circ} \text { de réplicas eficaces/ } \\
\text { eficientes/ pertinentes } \\
\text { por Tipos de Actividades } \\
\text { comunicativas de la lengua }\end{array}$} & \multicolumn{5}{|c|}{$\begin{array}{l}\text { \% de réplicas eficaces/ } \\
\text { eficientes/ pertinentes } \\
\text { por Tipos de Actividades } \\
\text { comunicativas de la lengua }\end{array}$} \\
\hline Z & $\begin{array}{l}\text { EO } \\
90\end{array}$ & $\begin{array}{l}C A \\
33\end{array}$ & $\begin{array}{l}\mathrm{EE} \\
20\end{array}$ & $\begin{array}{c}C L \\
3\end{array}$ & $\begin{array}{c}10 \\
1\end{array}$ & $\begin{array}{c}\text { EO } \\
14\end{array}$ & $\begin{array}{l}C A \\
10\end{array}$ & $\begin{array}{c}\mathrm{EE} \\
1\end{array}$ & $\begin{array}{c}C L \\
-\end{array}$ & $\begin{array}{c}10 \\
-\end{array}$ & $\begin{array}{c}\text { EO } \\
15 \%\end{array}$ & $\begin{array}{l}\text { CA } \\
31 \%\end{array}$ & $\begin{array}{l}E E \\
5 \%\end{array}$ & $\begin{array}{c}C L \\
-\end{array}$ & $\begin{array}{c}10 \\
-\end{array}$ \\
\hline $\mathrm{Y}$ & $\begin{array}{c}\text { EO } \\
3 \\
\end{array}$ & $\begin{array}{c}C A \\
3 \\
\end{array}$ & $\begin{array}{l}\mathrm{EE} \\
24 \\
\end{array}$ & $\begin{array}{c}\text { CAv } \\
21\end{array}$ & $\begin{array}{c}10 \\
3 \\
\end{array}$ & $\begin{array}{c}\text { EO } \\
-\end{array}$ & $\begin{array}{c}\text { CA } \\
- \\
\end{array}$ & $\begin{array}{c}\mathrm{EE} \\
5 \\
\end{array}$ & $\begin{array}{c}\text { CAv } \\
6 \\
\end{array}$ & $\begin{array}{c}10 \\
- \\
\end{array}$ & $\begin{array}{c}\text { EO } \\
- \\
\end{array}$ & $\begin{array}{c}\text { CA } \\
- \\
\end{array}$ & $\begin{array}{c}E E \\
20 \% \\
\end{array}$ & $\begin{array}{l}\text { CAv } \\
28 \% \\
\end{array}$ & $\begin{array}{c}10 \\
- \\
\end{array}$ \\
\hline$x$ & $\begin{array}{l}\text { EO } \\
22\end{array}$ & $\begin{array}{l}C A \\
27\end{array}$ & $\begin{array}{c}\text { EE } \\
- \\
\end{array}$ & $\begin{array}{c}C L \\
-\end{array}$ & $\begin{array}{c}10 \\
- \\
\end{array}$ & $\begin{array}{c}\text { EO } \\
8\end{array}$ & $\begin{array}{c}\text { CA } \\
8\end{array}$ & $\begin{array}{c}\mathrm{EE} \\
-\end{array}$ & $\begin{array}{c}\mathrm{CL} \\
-\end{array}$ & $\begin{array}{c}10 \\
-\end{array}$ & $\begin{array}{c}\text { EO } \\
36 \%\end{array}$ & $\begin{array}{c}C A \\
29 \%\end{array}$ & $\begin{array}{c}E E \\
-\end{array}$ & $\begin{array}{c}C L \\
-\end{array}$ & $\begin{array}{c}10 \\
-\end{array}$ \\
\hline W & $\begin{array}{c}\text { EO } \\
19\end{array}$ & $\begin{array}{c}C A \\
-\end{array}$ & $\begin{array}{l}\mathrm{EE} \\
16\end{array}$ & $\begin{array}{l}\mathrm{CL} \\
14\end{array}$ & $\begin{array}{l}10 \\
16\end{array}$ & $\begin{array}{c}\text { EO } \\
4\end{array}$ & $\begin{array}{c}C A \\
-\end{array}$ & $\begin{array}{c}\mathrm{EE} \\
4\end{array}$ & $\begin{array}{c}C L \\
6\end{array}$ & $\begin{array}{c}10 \\
2\end{array}$ & $\begin{array}{l}\text { EO } \\
21 \%\end{array}$ & $\begin{array}{c}C A \\
-\end{array}$ & $\begin{array}{c}E E \\
25 \%\end{array}$ & $\begin{array}{c}\mathrm{CL} \\
42 \%\end{array}$ & $\begin{array}{c}10 \\
33 \%\end{array}$ \\
\hline & & & & & & & & & & & $\begin{array}{l}\text { TOTAL } \\
72 \%\end{array}$ & $\begin{array}{l}\text { TOTAL } \\
60 \%\end{array}$ & $\begin{array}{c}\text { TOTAL } \\
50 \%\end{array}$ & $\begin{array}{l}\text { TOTAL } \\
70 \%\end{array}$ & $\begin{array}{c}\text { TOTAL } \\
33 \%\end{array}$ \\
\hline
\end{tabular}

\section{Interpretación de resultados}

Nos situamos ante los resultados obtenidos en una doble dimensión interpretativa: a) la global de los resultados que nos permiten formular inferencias de manera objetiva y sistemática; y b) la individual que, mediante una lectura en profundidad, nos permite vincular los resultados a la especificidad de cada aula y del profesorado responsable, formulando igualmente inferencias por una valoración cualitativa.

\section{a) Dimensión interpretativa global}

Las actividades relativas estrictamente a la oralidad (EO, CA, IO) arrojan valores totales porcentuales de réplicas eficaces, eficientes y pertinentes de alto grado de significatividad en el recurso a la mediación oral: EO $72 \%, C A 60 \%$ e $1033 \%$, teniendo en cuenta el número de actividades que se movilizaron, en número de 28,14 y 5 respectivamente.

b) Dimensión interpretativa individual

En el Aula Z las réplicas eficaces, eficientes y pertinentes de mediación oral que han tenido lugar en las actividades relativas estrictamente a la oralidad (EO y CA) suman un valor porcentual de $46 \%$, que revela, aun no habiéndose planificado actividades de 10 , la intensidad del recurso a la mediación oral.

Cabe inferir que, aun en el caso de la Profesora $Z$ con tan breve experiencia docente, los efectos de la mediación oral se han puesto de manifiesto en las réplicas registradas. Existe en este caso una sensibilización implícita por parte de esta profesora, por cuanto que si bien ha tomado conciencia de las directrices oficiales para el desarrollo del currículo de ILE, en el acto profesional de planificación (Roy, 1991), ha tomado decisiones centradas en adecuarse a la especificidad del aula y querer evitar conflictos comunicativos.

Estas interpretaciones se ven apoyadas por el posicionamiento metodológico explícito, manifestado por la Profesora Z, en el sentido de relegar a un segundo plano la realización de algunas actividades comunicativas de la lengua. 
En el Aula $Y$ no hemos podido identificar réplicas eficaces, eficientes y pertinentes en las actividades comunicativas de la lengua relativas estrictamente a la oralidad (EO, CA, IO), a pesar de que como aparece consignado más arriba, han sido realizadas seis. Aun habiendo conflictos comunicativos resueltos, no son consideradas por cuanto que no se ha recurrido a la mediación oral en la lengua objeto de aprendizaje (ILE).

La presencia masiva de la lengua de escolarización impide que cada alumno pueda aprovechar su bagaje cognitivo en su primera lengua/ cultura y así facilitar el aprendizaje, conforme a los planteamientos constructivistas (Tascón Trujillo, 2003).

En el caso de la Profesora $Y$, aun cuando posee una amplia experiencia docente, el hecho de haber observado el recurso demasiado frecuente a la traducción, cabría inferir que carece de sensibilización a los efectos beneficiosos del uso de la lengua objeto de aprendizaje y, en consecuencia, al uso de la mediación oral en ILE; o bien, que no domina suficientemente el ILE. De ahí, quizá, que sea la única que planifique la actividad Tipo CAv (no estrictamente relativa a la oralidad) que supone para ella movilizar lo paratextual.

Estas interpretaciones se ven apoyadas, asimismo, por el posicionamiento metodológico explícito manifestado por esta profesora, marcado por sus preconcepciones sobre las carencias lingüísticas de su alumnado, extensivas a todo el grupo.

En el Aula $X$ se alcanza un valor porcentual total de $65 \%$ en las réplicas eficaces, eficientes y pertinentes de mediación oral en las únicas actividades comunicativas de la lengua planificadas (EO, CA); y precisamente se trata de las estrictamente relativas a la oralidad.

Cabe inferir que, en el caso del Profesor X, su opción de relegar a un segundo plano la realización de otras actividades comunicativas de la lengua obedece, aun siendo poseedor de una experiencia docente más bien media, a un alto grado de sensibilización ante las eventuales limitaciones de su alumnado, pues se trata de un aula del primer nivel de Educación Primaria. Nivel para el que se ha establecido una primacía de la oralidad en el desarrollo curricular del ILE. Bien es cierto que el Profesor $X$ no se ha posicionado de manera explícita al respecto, pero podemos inferir que, tanto en el acto profesional de planificación como en el de intervención, ha optado por adecuarse a la especificidad del aula, al igual que sucede en el caso del Aula $Z$.

En el Aula $W$ las réplicas eficaces, eficientes y pertinentes de mediación oral en las actividades relativas estrictamente a la oralidad (EO, IO) suman un valor porcentual de $54 \%$, siendo la única aula en donde podemos identificar estas réplicas vinculadas a la actividad comunicativa de IO.

Ante estas consideraciones podemos inferir que aun siendo una profesora con experiencia media-baja docente, el hecho de ser nativa y de tratarse de un centro con proyecto bilingüe, posee un alto grado de sensibilización ante las expectativas generadas desde el punto de vista curricular.

\section{Conclusiones}

En función de los objetivos planteados, podemos concluir que:

1. De entre todas las actividades comunicativas de la lengua observadas, han sido las vinculadas más estrictamente con la oralidad, como son las de Comprensión auditiva y las de Expresión oral, las que: 
- de forma más intencional, han sido movilizadas en las aulas de ILE con alumnado extranjero, dando lugar a más réplicas con mayor efecto para la resolución o evitación de los conflictos comunicativos;

- indirectamente, más han mostrado que favorecen la participación del alumnado extranjero en el aula como instancia educativa y en el proceso de enseñanza/aprendizaje de ILE en sí mismo; y que a través de esa participación, constituyen un recurso con efectos añadidos sobre la integración social del alumnado extranjero en las aulas de ILE;

- en una perspectiva metodológica, ponen de manifiesto que constituyen un paso necesario previo a la promoción con mayor intensidad de las actividades de Interacción oral.

2. La capacidad de sensibilización a las potencialidades de la mediación oral debería ser abordada como componente competencial del perfil profesional del profesorado de ILE. En este orden de cosas, son de especial consideración como conocimientos que la sustentan los rasgos definitorios de la mediación, tales como la autonomía, el entendimiento, la participación, la anticipación y la predicción (Feuerstein, 1996). Constituye un valor añadido para los planes y modelos de desarrollo de competencias profesionales docentes, orientados a la obtención de cualificaciones profesionales y acreditación de competencias. Así lo consideran en sus aportaciones, conforme a las dinámicas europeas, Guillén Díaz y Barranco Izquierdo (2015) y Sanz Trigueros (2015).

\section{Referencias}

Arroyo González, R. (2004). Mediación didáctica. En F. Salvador Mata, J. L. Rodríguez Diéguez y A. Bolívar Botía (dirs.), Diccionario Enciclopédico de Didáctica, vol. II (pp. 224-225). Archidona (Málaga): Aljibe.

Bardin, L. (1986). El análisis de contenido. Madrid: Akal.

Bisquerra, R. (1989). Métodos de investigación educativa. Guía práctica. Barcelona: Ceac.

Consejo de Europa (2001). Marco Común Europeo de Referencia para las Lenguas: aprendizaje, enseñanza, evaluación. Estrasburgo: División de Políticas Lingüísticas. [2002, versión en español, por el Instituto Cervantes]. Disponible en: http://cvc.cervantes.es lobref/marco

De Ketele, J. M. y Gerard, F. M. (2007). La qualité et le pilotage du système éducatif. En Behrens, M. (ed.), La Qualité en éducation. Pour réfléchir à la formation de demain (pp. 19-38). Québec: Presses de I'Université du Québec. Disponible en: http://www.fmgerard.be/textes/pilotage.html

Feuerstein, R. (1996). La teoría de la modificabilidad estructural cognitiva. Educación cognitiva, 1, 31-75.

Guillén Díaz, C. y Barranco Izquierdo, N. (2015). Emergencia de nuevos desempeños docentes en función de la competencia en lenguas extranjeras y exigencia de cualificaciones específicas. En Actas Congreso Internacional Observal 2015. Cualificaciones profesionales y acreditación de competencias. Valladolid: Universidad de Valladolid (En prensa)

Kelly, M., et al. (2004). European Profile for Language Teacher Education. A Frame of Reference. Southampton: University of Southampton.

Krippendorff, K. (1990). Metodología de análisis de contenido. Teoría y práctica. Barcelona: Paidós. 
Legendre, R. (2005). Dictionnaire actuel de l'Éducation. Paris-Montréal: ESKA, Guérin.

Llorent García, V. J. \& López Azuaga, R. (2012). Demandas de la Formación del Profesorado. El desarrollo de la educación inclusiva en la Educación Secundaria Obligatoria. REIFOP, 15 (3). Disponible en: http://revistas.um.es/reifop/article/view /207611/166431

Martínez Agudo, J. de D. (2003). Hacia una enseñanza de lenguas extranjeras basada en el desarrollo de la interacción comunicativa. Didáctica (Lengua y Literatura), 15: 139-160.

Roy, D. (1991). Étude de l'importance des connaissances de l'enseignant et de l'influence des actes professionnels d'enseignement pour l'apprentissage au collégial. Quebec: Cégep de Rimouski.

Sanz Trigueros, F. J. (2015). Del Marco Europeo de las Cualificaciones al "European Profiling Grid" como un referencial de empleabilidad de los docentes. En Actas Congreso Internacional Observal 2015. Cualificaciones profesionales y acreditación de competencias. Valladolid: Universidad de Valladolid (En prensa)

Tascón Trujillo, C. (2003). La función constructivista de la Mediación: el mediador y el aprendizaje mediado. Anuario de Psicología, Filosofía y Sociología, 6: 117-130.

Trujillo Sáez, F. (2008). Plurilingüismo en el aula: las lenguas de los estudiantes. En I. Ballano (coord.). I Jornadas sobre Lenguas, Currículo y Alumnado Inmigrante. Bilbao: Publicaciones de la Universidad de Deusto (pp. 61-70).

Weil-Barais, A. et Resta-Schweitzer, M. (2008). Approche cognitive et développementale de la médiation en contexte d'enseignement-apprentissage. La nouvelle revue de l'adaptation et de la scolarisation, 42: 83-98. 